\title{
Cross Cultural Adaptation, Translation, Validation and Reliability Analysis of Gujarati Version of Hypertension Quality of Life Questionnaire (MINICHAL)
}

\author{
Yagnik Dave $^{1}$, Dinesh Sorani ${ }^{2}$, Mital Patel ${ }^{3}$ \\ ${ }^{1} \mathrm{PhD}$ Scholar, Saurashtra University \& Assistant Professor Marwadi University, Rajkot. Faculty of \\ Physiotherapy, Marwadi University, Morbi-National Highway, Rajkot, Gujarat, India. \\ ${ }^{2}$ Principal, Government Physiotherapy College, Jamnagar. \\ ${ }^{3}$ HOD Anatomy, Government Medical College, Jamnagar.
}

Corresponding Author: Yagnik Dave

\begin{abstract}
Introduction: The instruments used to measure QOL are useful methods of transforming subjective measurements into objective data that can be quantified and analyzed, and are also important for assessing the impact of health care interventions on patient's HRQOL. The uses of a self-administered scale in a specific localize/native language would make research and the clinical management more effective. It is advised that a self-reported measure is translated and adapted to the appropriate culture before it is used with subjects whose first language is not English. A Guajarati version of Quality of Life (MINICHAL) in essential hypertension would provide Guajarati community opportunity to communicate more effectively with their therapist. The availability of a self-administered scale in their native language makes clinical management and research more effective.

Methodology: In this observational study, 36 patients with essential hypertension from the Rajkot city included. MINICHAL-QOL was translated into Gujarati from English using Forward - Backward - Forward method. To ensure the face and content validity using group consensus method, each item was examined by group of 6 experts having mean experience of 8.78 years in the field of Physiotherapy.

Result: The total value of intra-class correlation coefficient of test-retest reliability was 0.988 . Reliability estimated by internal consistency estimated a Cronbach's alpha of 0.879 . The translated Gujarati version of MINICHAL-QOL demonstrated good reliability and validity. In validity specifically face validity and content validity suggests valid tool which can be applicable in essential hypertensive patients.

Conclusion: The results indicated that the Gujarati version of MINICHAL had been successfully translated from English to Gujarati. The translated Gujarati version of MINICHAL-QOL demonstrated good reliability and validity. So, it can be useful tools to measure quality of life in essential hypertensive patients specifically applicable for Gujarati population.
\end{abstract}

Keywords: Gujarati version, MINICHAL, Quality of Life, Reliability, Validity

\section{INTRODUCTION}

What is health? "The health can be defined as the state of complete physical, mental and social well-being than just the absence of disease or any abnormality." Therefore, a patient's perspective about his/her state of health is recognized as an important parameter for assessing health outcomes and the efficacy of an intervention.(1)

Essential hypertension remains a very important modifiable risk factor for 
cardiovascular disease (CVD) despite important advances in understanding of its pathophysiology and the availability of effective treatment strategies. In the hypertensive patients, high blood pressure can increases the risk of cardiovascular diseases for millions of people worldwide, and there is evidence that the problem is only getting worse. In the past decade, ageadjusted rates of stroke incidence have risen, and the slope of the age-adjusted rate of decline in coronary disease has levelled off. (2)

The overall burden of hypertensionrelated diseases is rapidly increasing in the developing world. Health Related Quality of Life (QOL) is a wide concept which can be affected in various ways by the person's physical health, mental state, the independence level, social relationships, personal beliefs, and their relationship to salient features with the environment. Health-related Quality of Life (HRQOL) is an emerging and a very important outcome in hypertension. (3)

The preservation of a patient's quality of life through prevention or treatment of illnesses has become more important in the health care system. One sign of the growing interest in health-related quality of life (HRQOL) is the increased number of publications on this humanistic and subjective outcome in most of the diseases and disorders. The Brazilian Guidelines for the Hypertension are very clear in defining the norms to be followed as to clinical results as well as outcomes in hypertension, but they do not set standards for the humanistic outcomes like HRQOL.(3)

The instruments/scales used to measure the quality of life are useful methods of transforming subjective measurements into objective data that can be quantified and analyzed and are also important for assessing the impact of health care interventions on a patient's HRQOL.

Validity is defined as the degree to which it measures what it is supposed to measure. The content/face validity, which indicates whether the questionnaire/scale makes sense to the patients and experts and whether all the important and relevant domains are included, was assessed by an expert panel. "Concurrent validity" is defined as the degree to which the operationalization correlates with other measures of the same construct that are measured at the same time. (4)

Reliability is the ability of a measurement to differentiate between subjects or objects. Reliability demonstrates homogeneity and reproducibility, in that, homogeneity consists of internal consistency of a scale while reproducibility consists of test-retest reliability of scores.(5)

The adapted instrument/scale allows a common measure to investigate the quality of life within different contexts which can be used in international and multi-centric studies, allowing comparisons to be made, besides saving time and financial resources and facilitates the research. The use of a self-reported questionnaire in a native language would make research and clinical management more effective. It is recommended that a self-reported measure/scale is translated and adapted to the appropriate English version, MINICHAL-HRQOL scale has been translated and adapted into Brazilian and Portuguese culture before it is used with subjects whose first language is not English.

MINICHAL is a multiple choice 16question questionnaire which is organized in 2 factors: first one is Mental Status (10 questions) and second one is Somatic Manifestations (6 questions), and separately one question to assess the patient's perception of how hypertension/high blood pressure and its treatment have influenced his/her quality of life. The answers in the domains are distributed on a Likert-type frequency scale, with four different answer options like from 0 (No, not at all) to 3 (Yes, very much). The maximum score for the Mental Status which includes 10 questions is 30 , whereas for the Somatic Manifestations domain which includes 6 questions and it is 18 . In this scale total 17 
questions includes, in which closer the result is 0 (zero), the better the quality of life. Question number 17, which assesses the patient's overall perception of own health, is also scored with the Likert scale but is not included in either of the two domains.(3)

Gujarati is an Indo-Aryan language native for Gujarat in India and it is spoken predominantly by the people of Gujarat. In India, it is the official language in the state of Gujarat, and the union territories of Daman and Diu and Dadra and Nagar Haveli. (6)

It is recommended that a measuring instrument validated and it must be reliable in a local language be adapted crossculturally to a new country, instead of developing a new tool to evaluate the same phenomenon. The adapted instrument must allows a common measure to investigate quality of life within different contexts which can be used in international and multi-centric studies, allowing comparisons to be made, besides saving time and financial resources. Translating a questionnaire/scale instead of formatting a new questionnaire allows comparisons of different populations, permits researchers to examine functional status across a broad spectrum of people, and permits the exchange of information across cultural and linguistic barriers. It is now widely recognized that questionnaires intended for use across the cultures must be translated perfectly and adapted culturally in order to maintain the content validity of the instrument. (7)

There is no reliable measure exists to measures Quality of Life (MINICHAL) in essential hypertensive patients in Gujarati language. It is an important tool for evaluation of the Quality of life in patient with essential hypertension. So, the purpose of this study was to translate the tool in Gujarati version from a humanistic point of view more and more becomes a part of the overall approach to health care, generating a need for reliable instruments to measure these parameters.
A Guajarati version of Quality of Life (MINICHAL) in essential hypertension would provide opportunity for Gujarati community to communicate more effectively with their therapist. The availability of a subjective tool/scale in their native language will make clinical management and research more effective.

\section{Aim \& Objectives of the study}

The main aims of the present study were to translate Quality of Life (MINICHAL) in Gujarati language and check the psychometric properties of the Quality of Life (MINICHAL). The objectives of this study were to test the face validity, content validity, internal consistency, test-retest reliability of Quality of Life (MINICHAL) in Gujarati speaking Essential Hypertensive patients.

\section{MATERIALS AND METHODS}

The study was conducted in Gujarat, from January-2021 to August-2021. For forward translation, a group of translators is usually included in the process. Therefore, Quality of Life (MINICHAL) translation was done by bilingual experts; two were related to subject and two were language experts with vast experience. Forward translations were reviewed by committee members and revisions in the few items were made according to their suggestions and comments.

After completion of the forward translation, a panel of 05 experts evaluated the different versions of forward translations. After this process, the draft was arranged for back-translation of Quality of Life questionnaire (MINICHAL). The members of the committee had familiarity with both cultures, the construct measured in the test, the basic principles along with the linguistic expertise, as suggested.

In backward translation from Gujarati to English, a bilingual expert who had not seen the original version of the test before translated the Gujarati version of Quality of Life MINICHAL (G-Quality-oflife questionnaire in Arterial Hypertension 
MINICHAL) back into English, the source language.

After the required modifications were done as per the suggestion of the expert committee, the scale was provided to an independent bilingual English language expert translator for backward translation.
The original English version of MINICHAL and newly formed MINICHAL were conceptually equivalent but some questions were culturally different. But it adheres to the sense of the original version. After this 3-phase process, one final scale/tool was developed. (Fig-1)

\section{G-Quality-of-life questionnaire in Arterial Hypertension (MINICHAL)}

\begin{tabular}{|c|c|c|c|c|c|}
\hline ६모 & છેલ્લા ૭ દ્વસ દરામંયાન. & \begin{tabular}{|l|} 
नll, \\
જરાય \\
નહંl. (0)
\end{tabular} & $\begin{array}{l}\text { छL, } \dot{\delta} \delta 5 \\
\text { अंशे. (૧) }\end{array}$ & 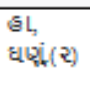 & 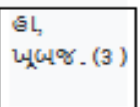 \\
\hline$q$ & ઘંં તમારા ઊધ ખરાબ થયેલા છે? & & & & \\
\hline २ & 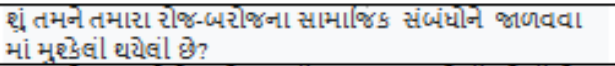 & & & & \\
\hline 3 & 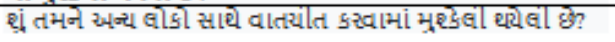 & & & & \\
\hline$\gamma$ & 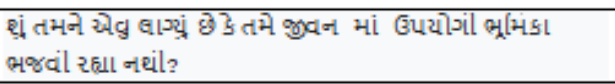 & & & & \\
\hline ૫ & 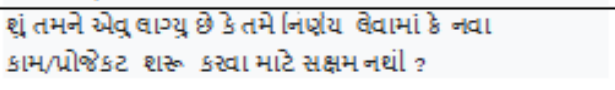 & & & & \\
\hline s & 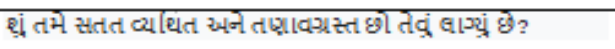 & & & & \\
\hline 9 & 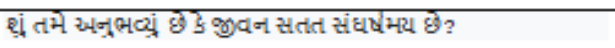 & & & & \\
\hline c & 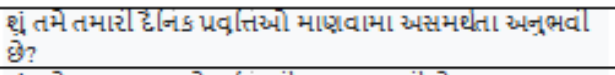 & & & & \\
\hline$e$ & 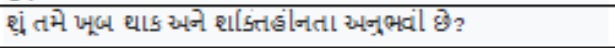 & & & & \\
\hline १० & 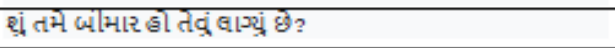 & & & & \\
\hline ११ & 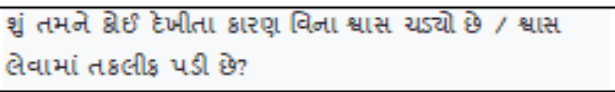 & & & & \\
\hline १२ & શ્રં તમારો પગનાં ધૂંટી માં સોજો આવેલ છે? & & & & \\
\hline १३ & $\begin{array}{l}\text { શ્રે તમે અનુભવ્યુ છે કે તમારેવારંવાર પેશાબ કરવા જવું પડી રહું } \\
\text { છે? }\end{array}$ & & & & \\
\hline १. & છંં તમારું મો સુકાઈ ગયું ્ઞેય તેવું અનુભવ્યું છે? & & & & \\
\hline १५ & 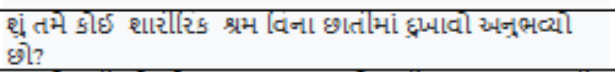 & & & & \\
\hline १९ & 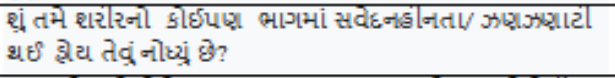 & & & & \\
\hline १ง & 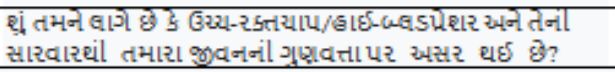 & & & & \\
\hline
\end{tabular}

Fig-1: G-Quality-of-life questionnaire in Arterial Hypertension MINICHAL

Participants: Native Gujarati patients with essential hypertension, both male and female age with 35 to 65 recruited for the study in Rajkot, Gujarat, India. Patients were excluded Secondary hypertension due to liver, heart, renal failure. Having disease of respiratory tract e.g. history of tuberculosis, chronic obstructive airway disease respiratory tract infections. Informed consent was taken from each patient before participation in written format. Ethical approval was not sought as this study was non-experimental in nature. To ensure the face and content validity using group consensus method, each item was examined by group of experts having mean experience of 8.78 years in the field of Physiotherapy. Each item of Gujarati version of MINICHAL was analyzed for content, meaning, wording, format, an ease of administration and scoring. Each item was scored by expert group as either accepted, rejected or accepted with modifications in the newly formed Gujarati version of MINICHAL scale.

Face validity: Face validity is a subjective assessment of whether the measure appears relevant to the ones to be measured. Face validity was assessed by asking one question to each patient, 'Do you think this scale is relevant to your condition?' The 
answer was noted in form of either 'YES' or 'NO'. Face validity of MINICHAL-QOL was established when all the 36 participants questioned about the relevance of the scale to their condition, all patients answered 'YES'. (8)

Content Validity: Content validity was assessed under the following headings:

1. Are the words in the translated Gujarati version of MINICHAL scale presented fluently and correctly as in the original version? For this answers from 6 expert panel members fall between 'Mostly Agree' to 'Strongly Agree'. (Average= 4.62)

2. Do the words and phrase in the translated Gujarati version of MINICHAL scale have the same semantic meaning compared with the original version? For this answers from 6 expert panel members fall between 'Mostly Agree' to 'Strongly Agree'. $($ Average $=4.72)$

3. Content relevance assessed by asking How the Gujarati version of MINICHAL scale statement is relevant to assessing Quality of life in essential hypertension patients? For this answers from 6 experts panel members fall between ' Mostly Agree' to 'Strongly Agree' (Average $=4.52$ )

4. Content representativeness was assessed by asking 'How well is the content (Item no. 1 to 17) of MINICHAL-QOL Gujarati scale is representing the entire scale in essential hypertension?' For this answers from 6 experts panel members falls between 'Good' to 'Very good' on a 5 point Likert scale (Average=4.5).(8)

Internal Consistency: Internal consistency of the MINICHAL- QOL was examined with Cronbach's alpha coefficient. Cronbach's alpha values range from 0 to 1 , where the values above 0.7 indicate adequate internal consistency for a scale.(8)

Test - Retest Reliability: Test-retest reliability of the questionnaire was undertaken by 36 subjects for the MINICHAL-QOL Gujarati version. Subjects completed the relevant questionnaire twice with an interval of 48 Hours to minimize any memory of previous answers and any variation in clinical status of essential hypertensive patients. Testretest was determined by Intra-class Correlation (ICC). ICCs can vary from 0 to 1 and values above 0.8 are considered as evidence of excellent reliability. (8)

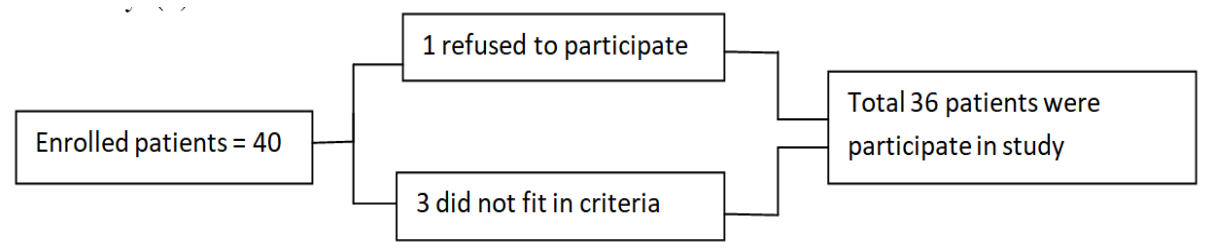

\section{Statistical Analysis:}

Descriptive statistics (Percentage, average, and Standard deviations) were used to describe demographic characteristics within the study. Analysis of reliability described in the research methods was conducted using SPSS statistical package (Version 20.0).

Statistical analysis: The data were checked for normality using the KolmogorovSmirnov test. Descriptive statistics like percentage, means, and standard deviations were used to describe demographic characteristics within the study. All analyses of reliability and validity described in the research methods were conducted using SPSS version 20.0 (IBM, Armonk, NY, USA) for Windows with a $95 \%$ confidence interval $(\mathrm{CI})$.

\section{RESULT}

A total 36 patients with essential hypertension (Male 22, Female 14) participated in the study. The demographic 
and clinical characteristics of the patients are presented in Table-1.

Table-1 Baseline characteristics of Patients

\begin{tabular}{|l|l|}
\hline Characteristics & Values \\
\hline Subjects (n) & 356 \\
\hline Male/Female & $22 / 14$ \\
\hline Age in Years- Mean (SD) & $41 \pm 4$ \\
\hline Systolic blood pressure & $148 \pm 4.86$ \\
\hline Diastolic blood pressure & $89 \pm 2.24$ \\
\hline
\end{tabular}

*The values expressed in table as mean and standard deviation.

The total score ranged between 6 and 46 for MINICHAL Quality of Life. In this study, $14(38.8 \%)$ of patients out of 36 with essential hypertensive patients were found to have a high level of affection in QOL. Test-Retest reliability of the MINICHAL Quality of Life analyzed on stable subjects $(\mathrm{n}=36)$ yielded excellent results with a confidence intervals (0.988) [Table 2]. The MINICHAL Quality of Life exhibited excellent internal consistency shown by a Cronbach's $\alpha$ value of 0.879 . [Table 3]

\section{Test retest reliability}

Table 2: Test retest reliability of G-Quality of life questionnaire in Arterial Hypertension MINICHAL

\begin{tabular}{|l|l|l|l|}
\hline Que number & R-value & Que number & R-value \\
\hline 1 & 1 & 10 & 1 \\
\hline 2 & 1 & 11 & 0.991 \\
\hline 3 & 0.989 & 12 & 0.939 \\
\hline 4 & 1 & 13 & 1 \\
\hline 5 & 0.984 & 14 & 0.989 \\
\hline 6 & 1 & 15 & 0.939 \\
\hline 7 & 0.991 & 16 & 1 \\
\hline 8 & 1 & 17 & 1 \\
\hline 9 & 0.984 & Average & 0.988 \\
\hline
\end{tabular}

\section{Internal consistency}

Table 3: Internal consistency of G-Quality of life questionnaire in Arterial Hypertension MINICHAL

\begin{tabular}{|l|l|l|l|}
\hline $\begin{array}{l}\text { Que } \\
\text { number }\end{array}$ & $\begin{array}{l}\text { Cronbach's } \\
\text { Alpha value }\end{array}$ & $\begin{array}{l}\text { Que } \\
\text { number }\end{array}$ & $\begin{array}{l}\text { Cronbach's } \\
\text { Alpha value }\end{array}$ \\
\hline 1 & 0.971 & 10 & 0.871 \\
\hline 2 & 0.833 & 11 & 0.973 \\
\hline 3 & 0.867 & 12 & 0.863 \\
\hline 4 & 0.901 & 13 & 0.811 \\
\hline 5 & 0.929 & 14 & 0.863 \\
\hline 6 & 0.863 & 15 & 0.833 \\
\hline 7 & 0.811 & 16 & 0.901 \\
\hline 8 & 0.823 & 17 & 0.901 \\
\hline 9 & 0.937 & Average & 0.879 \\
\hline
\end{tabular}

\section{DISCUSSION}

There is much emphasis today on using standardized and validated research instruments as these enable comparison of results both within and across countries. A patient reported outcome is an umbrella term that covers a whole range of potential types of measurement; it is used specifically to refer to measures that quantify a patient's state of health in terms of outcomes reported by patients. Thus, in a country like India, instruments like the MINICHAL-QOL have significant ethical implications for several reasons. Linguistically translated versions are not the same as those that have undergone cross-cultural adaptation; the latter being needed when the scale is used in another country, language and setting.(8)

For chronic disease like hypertension assessing QOL can help in evaluating the physical and psychosocial impact of the disease in affected individuals. It can also serve as important outcome measure for different kind of interventions whether it may pharmacological or nonpharmacological. According to a recent study, patients with hypertension have worse QOL. Mean score for MINICHAL was range 6 and 46 which was higher compared to previous study from Brazil suggesting poorer QOL of the study population.

In India, there are 22 officially recognized languages. Data in multi-centric studies are ultimately pooled and, thus, cross-cultural adaptation is necessary for each one of these languages if the pooled data are to be really relevant.(9)

The results showed that Gujarati version of MINICHAL used to be reliable and valuable tool for measuring QOL in essential hypertensive patients.

\section{CONCLUSION}

The results indicated that the Gujarati version of MINICHAL had been successfully translated from English to Gujarati. The translated Gujarati version of MINICHAL-QOL demonstrated good reliability and validity. So, it can be useful tools to measure quality of life in essential hypertensive patients specifically applicable for the Gujarati population. 
Acknowledgement: The authors would like to thank Dr. Jayeshbhai Parmar, Dr. Ashish Kakkad, Dr. Hemang Jani, Dr. Gauravgiri Gosai, Dr. Saloni Thaker, and Dr. Rahul chhatlani for providing their valuable inputs in the study.

\section{Contribution details:}

Concept \& design: Dr. Yagnik Dave

Definition of intellectual content: Dr. Dinesh Sorani

Literature search \& data acquisition: Dr Yagnik Dave

Data analysis \& statistical analysis: Dr Yagnik Dave

Manuscript preparation: Dr Yagnik Dave Manuscript editing: Dr. Dinesh Sorani Manuscript review: Dr Mital Patel

\section{Conflict of Interest: None}

\section{Source of Funding: None}

\section{Ethical Approval: Approved}

\section{REFERENCES}

1. Sinha R, Van Den Heuvel WA, Arokiasamy P. Validity and reliability of MOS short form health survey (SF-36) for use in India. Indian J Community Med. 2013;38(1):22-6.

2. Armstrong PW, Willerson JT. Clinical cardiology: New frontiers. Circulation. 1998;97(12):1107.

3. Schulz RB, Rossignoli P, Correr CJ, Fernández-Llimós F, De Toni PM. Validation of the short form of the spanish hypertension quality of life questionnaire (MINICHAL) for Portuguese (Brazil). Arq Bras Cardiol. 2008;90(2):139-44.

4. Rathod SR, Vyas N, Sorani DM. Validation of Gujarati Version of International Physical
Activity Questionnaire. Int J Heath Sci Res. 2018;8(1):122-8.

5. Shah S, Balaganapathy M. Reliability and validity study of the Gujarati version of the Oswestry Disability Index 2.1a. J Back Musculoskelet Rehabil. 2017;30(5):1103-9.

6. Dipmala S, Anjali B. Translation and validation of the Gujarati version of coronary revascularization outcome questionnaire in Percutaneous coronary intervention subjects. International Journal of Current Advanced Research. Volume 8; Issue 04 (G); April 2019; Page No.1851918522.

7. Bid, D. D., Soni, N. C., Rathod, P. V., Ramalingam, A. T., \& Sinha, R. K. (2016). Cross Cultural Adaptation, Reliability and Validity of Gujarati Version of Fear Avoidance Belief Questionnaire in Chronic Low Back Pain: Reliability and Validity of FABQ-G. National Journal of Integrated Research in Medicine, 7(6), 1-8.

8. Vaittianadane, Nambi G, Rathod P. Translation and validation of Guajarati version of WOMAC and Lequesne questionnaire in subjects with knee osteoarthritis. Int J Health Sci Res. 2018; 8(6):151-157.

9. Kathore V, More V. Study of effect of slow and deep breathing exercise on blood pressure among the patients with essential hypertension. MedPulse International Journal of Physiology. November 2019; 12(2): 24-28.

How to cite this article: Dave Y, Sorani D, Patel M. Cross cultural adaptation, translation, validation and reliability analysis of Gujarati version of hypertension quality of life questionnaire (MINICHAL). Int $J$ Health Sci Res. 2021; 11(10):10-16. DOI: https://doi.org/ 10.52403/ijhsr.20211003 